\title{
Honing in on hormone-sensitive neural targets for therapeutic intervention: mission impossible?
}

First draft submitted: 15 May 2017; Accepted for publication: 19 May 2017; Published online: 27 July 2017

Keywords: anxiety • depression $\bullet$ estradiol $\bullet$ estrogen $\bullet$ estrogen receptor $\bullet$ hormone therapy $\bullet$ hypothalamic paraventricular nucleus $\bullet$ oxytocin $\bullet$ raphe $\bullet$ serotonin

Stress-related neuropsychiatric disorders, such as depression and anxiety, are debilitating diseases that impose a great cost for patients, their families and society. Current first-line treatments for major depressive disorder and anxiety disorders target serotonergic neurotransmission, but the female-biased prevalence of these disorders has long suggested a role for hormones in the etiology and treatment of these diseases. Perhaps the most common hypothesis in this regard is that ovarian hormones promote resilience whereas declining hormone levels (e.g., after childbirth or menopause) increase susceptibility to depressive disorders. In fact, administering the potent estrogen, estradiol, as hormone therapy to perimenopausal women improves mood [1]. Moreover, polymorphisms in the genes for estrogen receptor (ER) $\alpha$ and the enzyme that metabolizes serotonin are both independently linked to depressive symptoms in menopausal women [2]. In addition to serotonin, times of endocrine flux may also contribute to vulnerability by causing dysregulation in oxytocin (OT) signaling. Methylation of the OT receptor (OTR) gene is associated with diagnoses of anxiety and/ or depression in older women [3] and postpartum women [4]. The relationship between OT and affect may be enhanced during these periods because estradiol regulates both OT and OTR through activation of ER- $\beta$ and $-\alpha$, respectively [5]. Together these data implicate gonadal steroid hormones, such as estradiol, and its cognate receptors as integral modulators of genes that regulate mood and anxiety. This further raises the possibility of harnessing hormone-sensitive brain networks as a therapeutic intervention for patients with diseases involving mood and affect. In this article, we will discuss two potential neural networks, the serotonergic system and the OTergic system, that could prove to be particularly useful targets for endocrine therapy.

Principle among antidepressant drug therapies are selective serotonin reuptake inhibitors (SSRIs), which prolong serotonergic signaling by increasing the time serotonin spends in synapses. Estrogenic hormones have been shown to mediate the efficacy of serotonin-related treatments. The anxiolytic effects of SSRI treatment are prevented by ovariectomy and are restored by estrogen replacement [6], and estrogen treatment augments the antidepressant effects of SSRIs in ovariectomized rats [7]. These results may be attributed to a serotonergic coordination of the anxiolytic and antidepressant effects of estrogen, as estradiol increases the expression of the serotonin transporter and serotonin-1B autoreceptor and blocking the serotonin-1B autoreceptor prevents the anxiolytic effects of estrogen [6,8]. Moreover, SSRI treatment itself can alter the endocrine state of patients. SSRIs are known to be endocrine disruptors and can reduce fertility, reduce androgens and increase the estrogen:androgen ratio in adrenocortical cell cultures, possibly by
Mary A Holschbach1, Amanda P Borrow ${ }^{1} \&$ Robert J Handa*,1

'Dept. Biomedical Sciences, Colorado State University, Fort Collins, CO 80523 , USA

*Author for correspondence:

Tel.: +1 9704915338

bhanda@colostate.edu 
increasing aromatase activity [9]. This further strengthens the need for understanding the relationship between the serotonin system and endocrine systems. Efficacious treatments are needed for distinct populations (e.g., women who hope to become mothers and perimenopausal women), and perhaps targeting identified hormone-sensitive neural circuits directly rather than providing systemic treatments could be an alternative strategy.

Serotonergic brain regions, such as the dorsal raphe, are exquisitely sensitive to hormones. Treating nonhuman primates with estradiol increases a number of genes and/or proteins that enhance serotonin neurotransmission, including $\mathrm{TPH} 2$, serotonin- $1 \mathrm{~A}$ receptors and serotonin- $1 \mathrm{~B}$ receptors $[10,11]$. ERs are expressed by serotonergic and nonserotonergic neurons of the dorsal raphe. However, estrogens are certainly not the only hormones that affect serotonergic neurotransmission. The neurons of the dorsal raphe also express high levels of glucocorticoid receptors, progesterone receptors and OTRs. Of these, the OTR is of particular interest. In fact, administering OT to healthy men has dramatic effects on serotonin signaling in the brain. By visualizing binding of a serotonin-1a receptor antagonist, Mottolese et al. showed that OT reduces extracellular serotonin in the dorsal raphe and several of its targets, including the amygdala. Administering OT to men increased between-region correlation in antagonist binding in the dorsal raphe and the amygdala, which suggests enhanced cross-talk between the two regions [12]. These findings are of particular importance because while both serotonin and OT were known to act in the amygdala to mediate behavioral responses to threatening stimuli, it was previously unknown whether the two systems operated independently or could interact.

In addition to being a potent regulator of serotonin signaling, the neural OT system could be a therapeutic target itself. While OT is classically known for its roles in reproduction [13] and social behavior [14], it also modulates mood and affect. Preclinical research has reported both anxiolytic [15] and antidepressive [16] effects of OT. Moreover, electroconvulsive therapy (ECT) increases neurophysin I, which is stimulated by estrogen and released stoichiometrically with OT, and the treatment-induced increase is greater in patients for whom ECT reduced depressive symptoms than those who were not relieved by ECT [17]. In contrast, a more recent report indicated that while depressed patients had lower levels of circulating OT than nonpatients, neither antidepressant drugs nor ECT had any effect on serum OT levels [18]. Patients with mood disorders may show abnormalities in OT function, as brain tissue from depressed human subjects indicates an elevation of OT mRNA in the paraventricular nucleus of the hypothalamus, a major site of OT synthesis [19] for oxytocin neurotransmission to forebrain sites that regulate behaviors [20]. This finding has also been observed in a preclinical model of depression known as the chronic stress model, as exposure to the chronic stress paradigm has induced an upregulation of OT mRNA within the paraventricular nucleus of the male rat [21]. Thus, it appears that affective disorders may be associated with dysregulated OT signaling.

The effects of OT on mood are believed to stem from this hormone's mediation of two distinct pathways: the serotonergic system and the hypothalamopituitary-adrenal (HPA) axis. Approximately, a fourth of serotonergic neurons in the dorsal raphe express OTR and, conversely, a third of OTR expressing neurons in the raphe are serotonergic. These neuroanatomical results are consistent with the observation that although OT acts in the raphe to reduce anxiety [22], this may not be due to OT action directly on serotonergic neurons because knocking out OTR from serotonergic cells had no effect on anxiety-like behavior [23]. These data suggest that OT's anxiolytic effects in the raphe may instead be coordinated by nonserotonergic neurons. The HPA axis, a major regulator of neuroendocrine stress responsivity, frequently shows dysregulation in patients with mood disorders [24], where depressed patients are unable to respond to glucocorticoids with negative feedback inhibition in a dexamethasone:corticotropin releasing hormone suppression test. While central OT largely has a suppressive effect on the HPA axis [25], the nature of OT's influence on this axis appears to be site-dependent since OT reportedly acts on the anterior pituitary gland to augment HPA axis activity through enhancing the secretogoue activity of corticotropin releasing hormone receptor CRFR1 [26]. Given this divergence in OT's effects on HPA axis activity, any treatment targeting the OT system to alleviate affective symptoms must consider its sites of action.

Despite the anti-anxiety and antidepressive qualities of OT, its clinical utility has been limited by its inability to penetrate the blood-brain barrier. Intranasal OT administration has shown potential in reducing symptoms of anxiety and, to a lesser extent, depression when delivered in high enough concentrations [27]. However, further investigation is still required to determine the efficacy of this treatment. Research using OTR agonists is currently limited, and while intracerebroventricular administration of the agonist carbetocin decreases anxiety-like behavior in the mouse, this compound has no effects on behavior when administered peripherally [28], providing further support for the hypothesis that the blood-brain barrier prevents 
peripheral OT manipulations from being effective. Thus, there is currently no known OTR agonist with therapeutic potential for the treatment of affective disorders. One promising means for indirectly activating the OT system is through treatment with estrogens. Estradiol upregulates expression of both OT and OTR [5]. If the OT system could be targeted by a circuit-specific endocrine therapy such as a selective ER modulator, it may be possible to induce a central OT response to peripherally administered treatments. Unfortunately, one caveat is that current compounds would not be feasible as they would likely cause off target effects in other brain regions.

Serotonin and OT are two of several neuromodulators with strong links to affect and mood disorders in humans and with robust literatures indicating involvement in nonhuman animal models of depression- and anxiety-related behaviors. Both of these neurotransmitter systems are sensitive to regulation by gonadal hormones, particularly estradiol. The development of approaches that can target ER activity with restricted effects to estrogen sensitive neural circuits in specific brain nuclei could open a new therapeutic avenue. Although recent advances have been able to deliver estradiol specifically to the brain [29], thereby bypassing

\section{References}

Papers of special note have been highlighted as:

- of interest; $\bullet$ of considerable interest

1 Schmidt PJ, Rubinow DR. Sex hormones and mood in the perimenopause. Ann. NY Acad. Sci. 1179, 70-85 (2009).

2 Rozycka A, Slopien R, Slopien A et al. The MAOA, COMT, MTHFR and ESR1 gene polymorphisms are associated with the risk of depression in menopausal women. Maturitas 84, 42-54 (2016).

3 Chagnon YC, Potvin O, Hudon C, Preville M. DNA methylation and single nucleotide variants in the brainderived neurotrophic factor (BDNF) and oxytocin receptor (OXTR) genes are associated with anxiety/depression in older women. Front. Genet. 6, 230 (2015).

4 Kimmel M, Clive M, Gispen F et al. Oxytocin receptor DNA methylation in postpartum depression. Psychoneuroendocrinology 69, 150-160 (2016).

5 Borrow AP, Handa RJ. Estrogen receptors modulation of anxiety-like behavior. Vitam. Horm. 103, 27-52 (2017).

6 Charoenphandhu J, Teerapornpuntakit J, Nuntapornsak A, Krishnamra N, Charoenphandhu N. Anxiety-like behaviors and expression of SERT and TPH in the dorsal raphe of estrogen- and fluoxetine-treated ovariectomized rats. Pharmacol. Biochem. Behav. 98(4), 503-510 (2011).

7 Ibrahim WW, Safar MM, Khattab MM, Agha AM. 17betaEstradiol augments antidepressant efficacy of escitalopram in ovariectomized rats: neuroprotective and serotonin reuptake transporter modulatory effects. Psychoneuroendocrinology 74 , potential peripheral side effects, brain region or circuit level specificity of such a targeted approach is beyond the reach of today's technology. The race to develop brain region or circuit-specific therapeutics could one day yield many beneficial treatment avenues for pharmaceutical exploration.

\section{Author contributions}

MA Holschbach and AP Borrow wrote the article with support and assistance from RJ Handa.

\section{Financial \& competing interests disclosure}

Funding from NIH via R01 NS039951 and MH082900 supported this work. The authors have no other relevant affiliations or financial involvement with any organization or entity with a financial interest in or financial conflict with the subject matter or materials discussed in the manuscript apart from those disclosed.

No writing assistance was utilized in the production of this manuscript.

\section{Open access}

This work is licensed under the Creative Commons Attribution 4.0 License. To view a copy of this license, visit http://creativecommons.org/licenses/by/4.0/

\section{0-250 (2016).}

8 Donner N, Handa RJ. Estrogen receptor beta regulates the expression of tryptophan-hydroxylase 2 mRNA within serotonergic neurons of the rat dorsal raphe nuclei. Neuroscience 163(2), 705-718 (2009).

9 Hansen CH, Larsen LW, Sorensen AM, Halling-Sorensen B, Styrishave B. The six most widely used selective serotonin reuptake inhibitors decrease androgens and increase estrogens in the H295R cell line. Toxicol. In vitro 41, 1-11 (2017).

10 Bethea CL, Lu NZ, Gundlah C, Streicher JM. Diverse actions of ovarian steroids in the serotonin neural system. Front. Neuroendocrinol. 23(1), 41-100 (2002).

11 Bethea CL, Kohama SG, Reddy AP, Urbanski HF. Ovarian steroids regulate gene expression in the dorsal raphe of old female macaques. Neurobiol. Aging 37, 179-191 (2016).

12 Mottolese R, Redoute J, Costes N, Le Bars D, Sirigu A. Switching brain serotonin with oxytocin. Proc. Natl Acad. Sci. USA 111(23), 8637-8642 (2014).

13 Borrow AP, Cameron NM. The role of oxytocin in mating and pregnancy. Horm. Behav. 61(3), 266-276 (2012).

14 Lukas M, Toth I, Reber SO, Slattery DA, Veenema AH, Neumann ID. The neuropeptide oxytocin facilitates prosocial behavior and prevents social avoidance in rats and mice. Neuropsychopharmacology 36(11), 2159-2168 (2011).

15 Bale TL, Davis AM, Auger AP, Dorsa DM, McCarthy MM. CNS region-specific oxytocin receptor expression: importance in regulation of anxiety and sex behavior. $J$. Neurosci. 21(7), 2546-2552 (2001). 
16 Yan Y, Wang Y-L, Su Z et al. Effect of oxytocin on the behavioral activity in the behavioral despair depression rat model. Neuropeptides 48(2), 83-89 (2014).

17 Scott AI, Whalley LJ, Bennie J, Bowler G. Oestrogenstimulated neurophysin and outcome after electroconvulsive therapy. Lancet 1(8495), 1411-1414 (1986).

18 Ozsoy S, Esel E, Kula M. Serum oxytocin levels in patients with depression and the effects of gender and antidepressant treatment. Psychiatry Res. 169(3), 249-252 (2009).

19 Meynen G, Unmehopa UA, Hofman MA, Swaab DF, Hoogendijk WJG. Hypothalamic oxytocin mRNA expression and melancholic depression. Mol. Psychiatry 12(2), 118-119 (2007).

20 Knobloch HS, Charlet A, Hoffmann LC et al. Evoked axonal oxytocin release in the central amygdala attenuates fear response. Neuron 73(3), 553-566 (2012).

21 Babygirija R, Bulbul M, Yoshimoto S, Ludwig K, Takahashi T. Central and peripheral release of oxytocin following chronic homotypic stress in rats. Auton. Neurosci. 167(1-2), 56-60 (2012).

22 Yoshida M, Takayanagi Y, Inoue $\mathrm{K}$ et al. Evidence that oxytocin exerts anxiolytic effects via oxytocin receptor expressed in serotonergic neurons in mice. J. Neurosci. 29(7), 2259-2271 (2009).

23 Pagani JH, Williams Avram S, Cui Z et al. Raphe serotonin neuron-specific oxytocin receptor knockout reduces aggression without affecting anxiety-like behavior in male mice only. Genes Brain Behav. 14(2), 167-176 (2015).

24 Watson S, Mackin P. HPA axis function in mood disorders. Psychiatry 5(5), 166-170 (2006).

25 Neumann ID, Krömer SA, Toschi N, Ebner K. Brain oxytocin inhibits the (re) activity of the hypothalamopituitary-adrenal axis in male rats: involvement of hypothalamic and limbic brain regions. Regul. Pept. 96(1-2), 31-38 (2000).

26 Gibbs D, Vale W, Rivier J, Yen S. Oxytocin potentiates the ACTH-releasing activity of CRF (41) but not vasopressin. Life Sci. 34(23), 2245-2249 (1984).

27 Hofmann SG, Fang A, Brager DN. Effect of intranasal oxytocin administration on psychiatric symptoms: a metaanalysis of placebo-controlled studies. Psychiatry Res. 228(3), 708-714 (2015).

28 Mak P, Broussard C, Vacy K, Broadbear JH. Modulation of anxiety behavior in the elevated plus maze using peptidic oxytocin and vasopressin receptor ligands in the rat. $J$. Psychopharmacol. 26(4), 532-542 (2012).

29 Prokai L, Nguyen V, Szarka S et al. The prodrug DHED selectively delivers $17 \beta$-estradiol to the brain for treating estrogen-responsive disorders. Sci. Transl. Med. 7(297), 297ra113 (2015). 